\title{
The Role of Digital Biomarkers in Cancer Research and Patient Care
}

\author{
Severin Rodler ${ }^{1 *}$, Gerald Schulz ${ }^{1}$, Alexander Buchner ${ }^{1}$, Christian Stief ${ }^{1}$, Michael Staehler ${ }^{1}$ and Jozefina \\ Casuscelli $^{1}$ \\ Department of Urology, Munich, Germany \\ *Corresponding author: Severin Rodler, Department of Urology, Munich, Germany
}

\begin{tabular}{|c|c|}
\hline ARTICLE INFO & ABSTRACT \\
\hline Received: 豐 April 19, 2019 & Classical biomarkers have been traditionally used to evaluate changes in the \\
\hline Published: April 26, 2019 & $\begin{array}{l}\text { homeostasis of the human body. With the emergence of digital technologies, new } \\
\text { opportunities have come up to continuously monitor patients during the course of illness }\end{array}$ \\
\hline $\begin{array}{l}\text { Citation: Severin R, Gerald S, Alexan- } \\
\text { der B, Christian S, Michael S and Joze- } \\
\text { fina C. The Role of Digital Biomarkers } \\
\text { in Cancer Research and Patient Care. } \\
\text { Biomed J Sci \& Tech Res 17(3)-2019. }\end{array}$ & $\begin{array}{l}\text { and therapy. Improved computational methods have enabled researchers to track and } \\
\text { analyze patient's health related data. While previous works have focused on the role of } \\
\text { digital biomarkers in cardiovascular and neurological diseases, we aim to address the role } \\
\text { of digital biomarkers in cancer research. Therefore, we provide a comprehensive review } \\
\text { on the use of digital biomarkers in clinical trials and patient care, regulatory environment } \\
\text { and future directions in this promising field. }\end{array}$ \\
\hline
\end{tabular}

BJSTR. MS.ID.003009.

Keywords: Digital Biomarker; Cancer; Wearable; Machine Learning; Digital Pathology; Clinical Trials

Abbreviations: EMR: Electronic Medical Record; FDA: Food and Drug Administration; SaMD: Software as a medical device; SiMD: Software in a Medical Device

\section{Introduction}

The impact of digital technologies on clinical trials and patient care is rising [1]. As in many other fields the major drivers for the wide adoption of digital technologies in health care are quick scalability and constantly decreasing costs [2]. In addition, this development is supported by improving computational power and new approaches to analyze great amounts of data by machine learning [3]. Biomarkers have traditionally been characterized as single time point assessments used to generate objectively measureable indicators of physiological changes of the human body. Biomarkers are currently employed in clinical trials [4] and influence clinical decision making [5]. Digital biomarkers are a emerging class of biomarkers that source their data from digital sensors or computational tools [6]. They can be composed of multiple hardware and software components and allow a continuous and remote assessment of patients [7]. So far, the impact of digital biomarkers on cancer research has not been intensively studied. Therefore we aim to provide an overview of the most important developments, regulatory burdens and future directions of digital biomarkers.

\section{FDA Regulation}

Digital biomarkers are regulated by the Food and Drug Administration (FDA) under the device section [8]. The FDA has undertaken significant efforts to deal with the upcoming new categories of medical devices and drugs aimed to track patient's health and disease. Traditionally, software was considered to be part of a hardware component and was classified as Software in a medical device (SiMD). But in light of the new technological developments, software can stand alone and is termed Software as a medical device (SaMD). To speed up market entry for new software the FDA has created a "precertification program". Therefore, rather than seeking for approval by the FDA, companies can register every new software or software update and undergo a precertification process to quickly release software and updates on a regular base. Amongst those companies are major tech corporations as Apple, Fitbit, Samsung and Verily, but also pharmaceutical companies as Johnson \& Johnson and Roche as well as Tidepool, Pear Therapeutics and Phosphorus [9]. 


\section{Current Digital Biomarker Landscape in Cancer Research and Patient Care}

Digital biomarkers can be generated from various sources like body sensors, image processing or from health platforms and electronic medical records (EMR). The use of wearable body sensors, termed wearables, is not only widespread in healthy people, but is also increasingly employed in cancer trials to track the physical activity, vital parameters and sleeping profiles [10]. In fact, wearables play an important role in oncology as they allow physicians to track their patients in a real world setting. So far, most clinical trials assessing activity have focused on breast and lung cancer patients [11]. Recently, it could be demonstrated that wearables can predicted performance scores in patients with advanced malignancies [12]. Ingestible sensors on the other hand aim to transmit time medication was taken as well as data on physiological changes of the digestive system. They can potentially be used to control therapy adherence and although they have not entered clinical routine in oncology first trials are ongoing [13]

Image processing and analysis performed by advanced analytic tools such as machine learning can also generate innovative digital biomarkers. In radiology, for instance, image recognition, followed by analysis is currently changing clinical routine in areas such as stroke diagnostics [14]. Further, first attempts are being undertaken in breast cancer screening using mammography images [15]. In pathology new biomarkers are being tested to determine the PD-L1 expression in cancer tissue [16]. In dermatology cancer diagnostics rely mostly on the morphology of lesions detected by dermatologists. However, a newly developed neuronal network can predict the malignancy potential of skin lesions more efficiently than trained dermatologists [17].

Health platforms and electronic medical records are the third important source for digital biomarkers. However, a major obstacle to access and analyze data from medical records is that this data is mostly not electronically accessible. The company Flatiron Health for instance has overcome this hurdle by capturing structured and unstructured medical data from oncologic patients. This method is able to generate predictive scores from electronic health record [18].

\section{Discussion}

Digital biomarkers are currently changing cancer research and start to get adopted in clinical decision-making. They can derive from body sensors, image processing or health platforms and electronic records and will have a substantial impact on clinical trials and patient management. Remote control of patients seems to be an interesting feature with wearables. In this case, biomarkers will not be limited to single-point measurements as traditionally performed during hospital visits, but will provide continuous measurements. Validation of biomarkers that rely on algorithms created by machine learning will remain a challenge. Since they can rely on a so called "blackbox" approach it is unclear what data the algorithms actually analyzes [19]. In addition, transferring biomarkers tested in certain subgroups to normal patient collectives can lead to wrong results.
As in traditional trials the information an algorithm generates from a training cohort is not necessarily true for the average patient population. Machine learning based biomarkers are trained by a training set of data, but the data can be biased as well and thereby the biomarker [20]. Therefore, a continuous validation process and post marketing studies might be required rather than a single time point validation.

\section{Conclusion}

The impact of digital biomarkers on cancer research and patient care is rising. However, novel technologies require new validation methods and regulatory burdens remain an issue that has to be addressed thoroughly by the approval authorities. Potentially, digital biomarkers will deliver more accurate diagnostic and prognostic markers for cancer patients. This will be possible alongside with costs reduction for the healthcare system and increasing access of patients to a high quality of care.

\section{References}

1. Clay I (2017) Impact of Digital Technologies on Novel Endpoint Capture in Clinical Trials. Clinical pharmacology and therapeutics 102(6): 912913.

2. Murray E, Hekler EB, Andersson G, Collins LM, Doherty A, et al. (2016) Evaluating Digital Health Interventions: Key Questions and Approaches. American journal of preventive medicine 51(5): 843-851.

3. Panch T, Szolovits P, Atun R (2018) Artificial intelligence, machine learning and health systems. Journal of global health 8(2): 020303.

4. Mandrekar SJ, Sargent DJ (2009) Clinical trial designs for predictive biomarker validation: theoretical considerations and practical challenges. Journal of clinical oncology 27(24): 4027-4034.

5. Selleck MJ, Senthil M, Wall NR (2017) Making Meaningful Clinical Use of Biomarkers. Biomarker insights 12: 1177271917715236.

6. Byrom B, Watson C, Doll H, Stephen Joel Coons, Sonya Eremenco, et al. (2018) Selection of and Evidentiary Considerations for Wearable Devices and Their Measurements for Use in Regulatory Decision Making: Recommendations from the ePRO Consortium. Value in health 21(6): 631-639.

7. Coravos A, Khozin S, Mandl KD (2019) Developing and adopting safe and effective digital biomarkers to improve patient outcomes. npj Digital Medicine.

8. Shuren J, Patel B, Gottlieb S (2018) FDA Regulation of Mobile Medical Apps. Jama 320: 337-338.

9. Administration USFaD. Digital Health Software Precertification (PreCert) Program.

10. Piwek L, Ellis DA, Andrews S, Joinson A (2016) The Rise of Consumer Health Wearables: Promises and Barriers. PLoS medicine 13: e1001953.

11. Gresham G, Schrack J, Gresham LM, Shinde AM, Hendifar AE, et al. (2018) Wearable activity monitors in oncology trials: Current use of an emerging technology. Contemporary clinical trials 64: 13-21.

12. Gresham G, Hendifar AE, Spiegel B, Elad Neeman, Richard Tuli, et al. (2018) Wearable activity monitors to assess performance status and predict clinical outcomes in advanced cancer patients. npj Digital Medicine.

13. (2019) Proteus digital health. Proteus Digital Health® Launches Digital Oncology Medicines to Improve Patient Outcomes.

14. (2018) FDA approves stroke-detecting AI software. Nature biotechnology 36: 290 . 
15. Rodriguez Ruiz A, Lang K, Gubern Merida A, Broeders M, Gennaro G, et al. (2019) Stand-Alone Artificial Intelligence for Breast Cancer Detection in Mammography: Comparison With 101 Radiologists. Journal of the National Cancer Institute.

16. Kapil A, Meier A, Zuraw A, Steele KE, Rebelatto MC, et al. (2018) Deep Semi Supervised Generative Learning for Automated Tumor Proportion Scoring on NSCLC Tissue Needle Biopsies. Scientific reports 8(1): 17343.

17. Esteva A, Kuprel B, Novoa RA, Justin Ko, Susan M. Swetter, et al. (2017) Dermatologist-level classification of skin cancer with deep neural networks. Nature 542: 115-118.

\section{ISSN: 2574-1241}

DOI: 10.26717/BJSTR.2019.17.003009

Severin Rodler. Biomed J Sci \& Tech Res

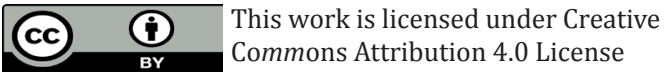

Submission Link: https://biomedres.us/submit-manuscript.php
18. Backenroth D, Haimson JD, J MN, Baxi SS (2019) Development and initial validation of a prognostic score based on structured data from EHRs. In: Proceedings of the 110th Annual Meeting of the American Association for Cancer Research,GA. Philadelphia (PA): AACR.

19. Char DS, Shah NH, Magnus D (2018) Implementing Machine Learning in Health Care - Addressing Ethical Challenges. The New England journal of medicine 378(11): 981-983.

20. Gianfrancesco MA, Tamang S, Yazdany J, Schmajuk G (2018) Potential Biases in Machine Learning Algorithms Using Electronic Health Record Data. JAMA internal medicine 178(11): 1544-1547.

BIOMEDICAL
RESEARCHES $\quad \begin{aligned} & \text { Assets of Publishing with us } \\ & \text { - Global archiving of articles }\end{aligned}$

